\title{
Mango Leaf Ailment Detection using Neural Network Ensemble and Support Vector Machine
}

\author{
Nabodip Sutrodhor, Molla Rashied Hussein, Md. Firoz Mridha, Prokash Karmokar, Tasrifa \\ Nur \\ Department of Computer Science and Engineering \\ University of Asia Pacific \\ Dhaka, Bangladesh
}

\begin{abstract}
This paper presents a Neural Network Ensemble (NNE) for Mango Leaf Ailment Detection (MLAD) system. At first, the images of Mango leaves were cropped, then were resized and converted to their value of threshold. After that, the feature extraction methodology was applied. For pattern recognition, NNE and SVM were used. Subsequently, test images of affected leaves were uploaded to the system and then were matched to the trained ailments. The training data and test data were crossvalidated to sustain equilibrium among over-fitting and underfitting issues.
\end{abstract}

\section{Keywords}

Neural Network Ensemble; Pattern Recognition; Support Vector Machine; Mango Leaf Ailment Detection; Image Processing; Automated System

\section{INTRODUCTION}

The fruit named Mango (Mangifera indica) was originated in the greater region of South Asia around 2000 BCE. Subsequently, the Mango has gradually been promulgated around the world, particularly by the Portuguese explorers. According to the scientific classification, the Mango is the member of Anacardiaceae family having Mangifera as genus and indica as species. The Mango is also renowned as the royal or king fruit in Asia [1].

The dominant adversity of growing Mango is the ailments in plants, particularly in the leaves, which curtails its production and downgrades its quality. Therefore, timely diagnosis of ailments is very critical. However, the ocular symptom for each ailment slightly differs from another and hence manual diagnosis becomes very challenging [2]. Several distinct approaches can be employed for detecting these ailments and most conventional are Artificial Neural Networks (ANNs) and Support Vector Machines (SVMs) [7].

In Agriculture sector in Bangladesh i.e. South Asia, technological advancements are getting pragmatic gradually [9]. Integrating Information and Communications Technology (ICT) with Agriculture inspires our inception of an automated system, namely, Mango Leaf Ailment Detection (MLAD). Several research works have been executed on detecting rice ailments, citrus ailments, wheat leaf ailments, betel vine plant ailments and other crop ailments. However, in case of MLAD, there is not enough research work to be performed to make it an efficient methodology [6]. Therefore, this research work contributes to the growth of MLAD, which will not only benefit the Mango industry of Bangladesh, but also the industry of the South Asia and other scattered regions that produce Mango in the world.

Breadth of this paper confines to the foremost four kinds of Mango leaf ailments. They are, namely, Scab (fungus), Anthracnose (fungus), Red Rust (Cephaleuros virescens, an algal plant pathogen) and Sooty Mold (caused by mealy bug, scale insect and hopper). Each ailment contrasts with another by the color it shows after affecting, shape of affected area, type of ailment medium (virus/fungus/algae/insect) and so on.

The organization of this paper is as follows: in section 2, the literature review is discussed, section 3 narrates the detailed methodology of MLAD, and section 4 depicts the result of the work being executed and its analysis. Finally, section 5 sketches out the conclusion along with some proposition on the research work to be pursued by fellow researchers in coming future.

\section{LITERATURE REVIEW}

Tzionas et al. [5] conducted a detailed analysis on a system that exhibits similar size orientation with respect to the samples. The system is capable of functioning appropriately even with leaves samples that are deformed due to drought or holes drilled in them. A significantly high classification ratio of $99 \%$ was obtained, even for the classification of deformed leaves.

Tian et al. [15] conducted another analysis on SVM-based Multiple Classifier System (MCS) for pattern recognition of wheat leaf ailments. The proposed system made use of stacked generalization structure to combine the classification decisions obtained from three kinds of SVM-based classifiers. Three different feature sets including color features, texture features and shape features are used as training sets for three corresponding classifiers. Initially, these dissimilar feature sets are categorized by the classifiers from low-level MCS to various corresponding mid-level categories, which are partly depicted by the symptom of crop ailments on the basis of plant pathology facts. Afterward, the mid-level features are extracted from these mid-categories produced from low-level classifiers. Lastly, high level SVMs were trained and errors made by the color, texture and shape were corrected for better recognition. In contrast to the previous classifiers for wheat leaf ailments, the proposed technique demonstrated better recognition.

Sladojevic et al. [7] came up with a new approach for the development of plant ailment recognition model using deep convolutional networks on the basis of leaf image classification. The authors discussed the essential steps required for implementing ailment recognition. Images were collected to create database which were assessed by agricultural experts. The developed model can identify 13 types of plant ailments out of healthy leaves along with the ability to distinguish plant leaves from their surroundings. A deep learning framework, Café developed by Berkley Vision and Learning Centre, was used to perform the deep CNN training. The experimental results on the developed model achieved precision in between $91 \%$ and $98 \%$. For separate class tests, the precision was found to be on average of $96.3 \%$.

Over the past decades, there have been many research works executed on incepting and developing of leaf ailment detection while producing Rice, Wheat, Betel-Vine and others. However, for MLAD, there has not been much work done, especially in 
Bangladesh. This paper aims to introduce and develop MLAD using NNE and SVM.

\section{METHODOLOGY}

The quality inspection of leaves consists of two main aspects, internal and external examinations. The internal quality inspection is usually achieved by human sensory, smoking test or chemical analysis, whereas the external quality inspection is mainly achieved through human vision. A number of reasons exist for using NNE. NNE offers much better generalization capability in contrast with a single monolithic $\mathrm{NN}$ in the same problem domain. In addition, in spite of having under-fitting and over-fitting problems, NNE works well in comparison with an individual NN due to its involvement in data re-sampling. In various cases, additional information is available which cannot be given to the same $\mathrm{NN}$ tackling the core information. The additional information can be trained separately before combination.

Among various NNE techniques, SVM has been employed in this work to develop MLAD. The SVM technique offers efficient feature extraction technique which allows MLAD to achieve faster training and better recognition performance. Figure 1 shows the analytical approach for Mango Leaf Ailment Detection (MLAD).

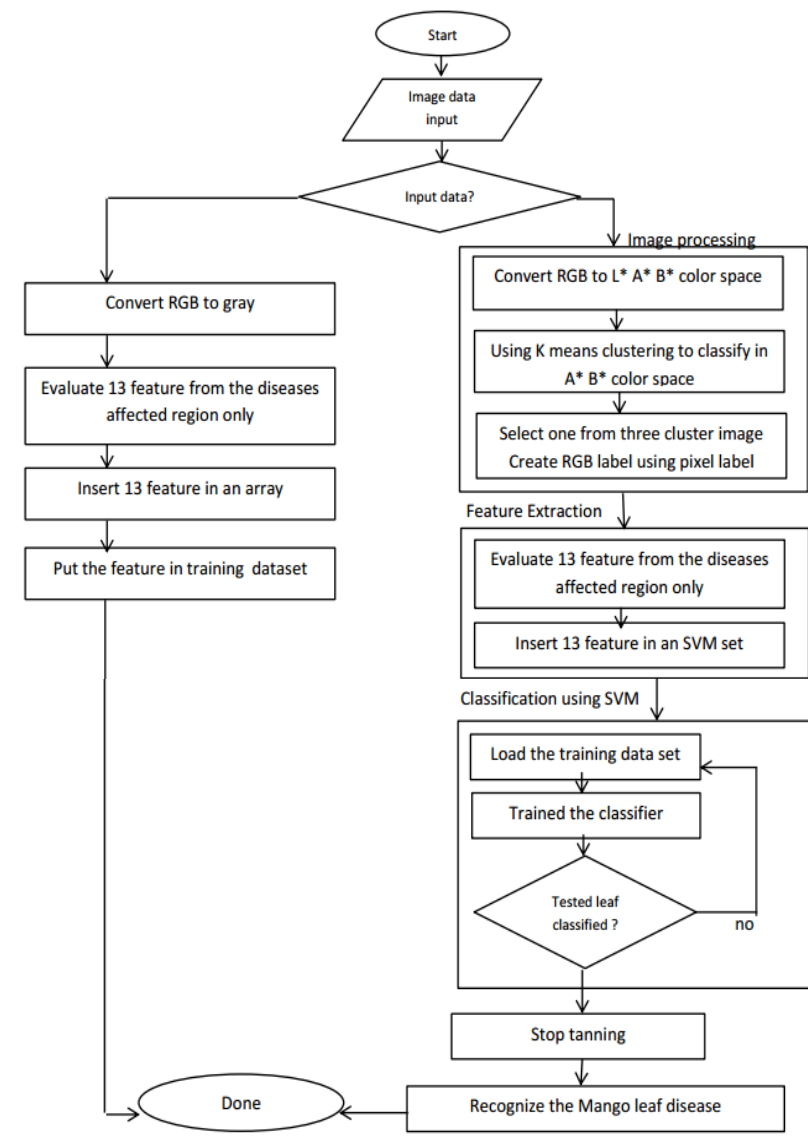

Figure 1: Analytical approach of MLAD

The following Figure shows an ailment free Mango leaf. This kind of leaf is not affected by any kind of ailment; hence data taken from this type can be referred as standard training data. When an image of an ailed leaf will be inserted into the system, the new data will be compared with these normal data from ailment free leaf.

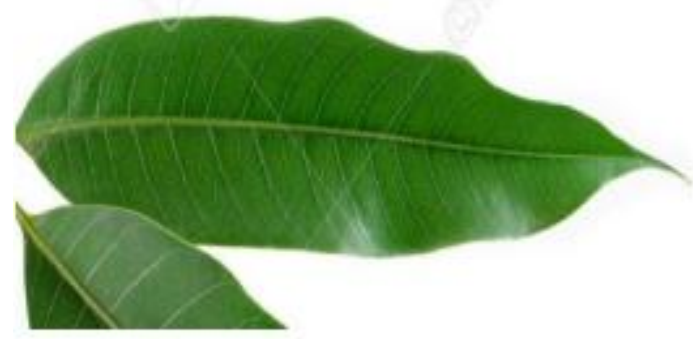

Figure 2: Ailment-free Mango Leaf

Figure 3 shows a Scab affected Mango leaf. Insects get attracted to this kind of leaf easily and dry it up.

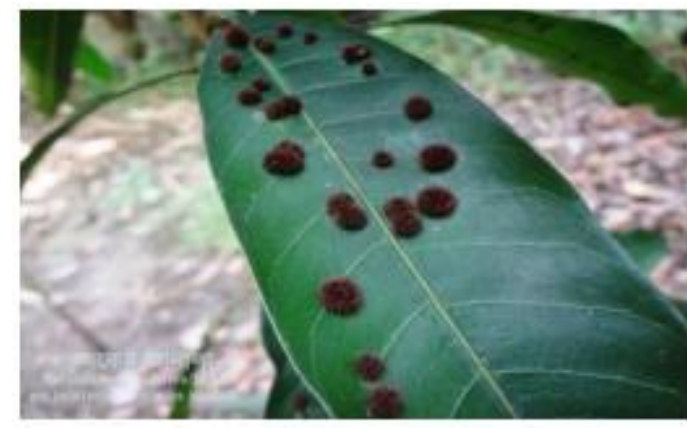

Figure 3: Scab (fungus) affected leaf

Figure 4 depicts Anthracnose, which affects leaves, stems and fruits and makes the leaves yellow and eventually brown. Also, the corner of the leaf gets dried up.

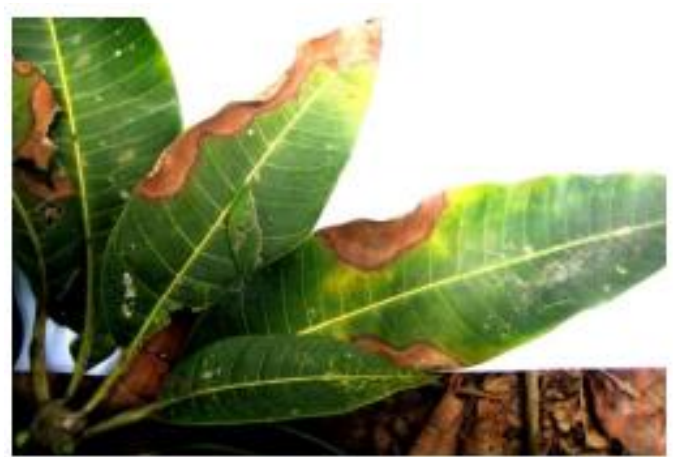

Figure 4: Anthracnose (fungus) affected leaf

Figure 5 illustrates Mango's Sooty Mold ailment can be observed in the presence of ants or hoppers or mealy bugs. They jointly attack the branches and their leaves and damage eventually in a large scale.

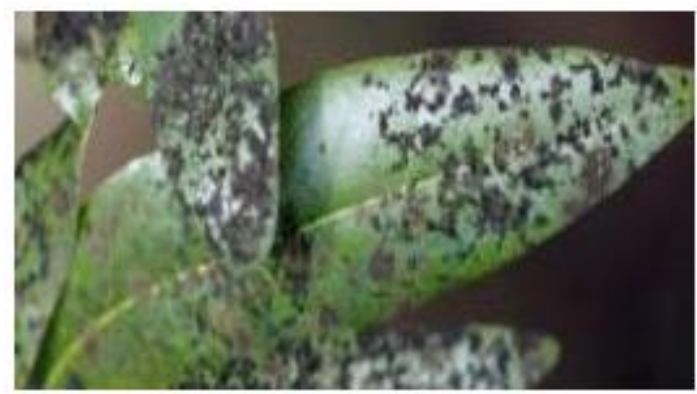

Figure 5: Sooty Mold (caused by mealy bug, scale insect and hopper)

Another kind of ailment on Mango leaf causes red rust spots in the trunk due to green algae attack. Figure 6 shows such red rust affected Mango leaf. 


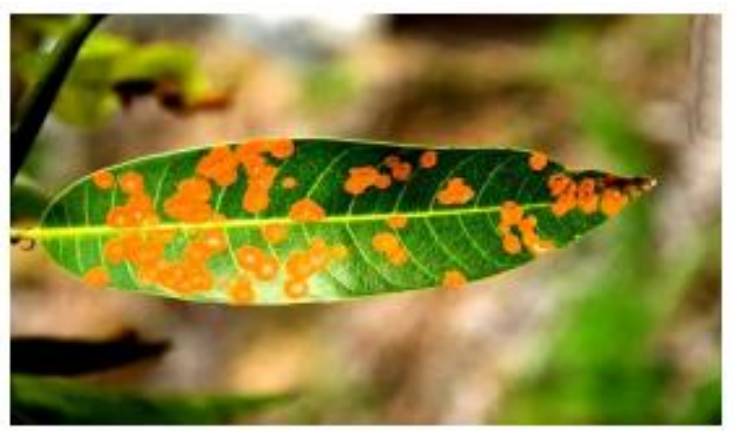

Figure 6: Red Rust (Cephaleuros virescens, an $\square$ algal plant pathogen) affected leaf

To carry out the detection process, the input data (image of leaves) are classified to identify the ailment. For recognition purpose, we have used SVM classification technique. Firstly, we have taken the image from current directory. To obtain better output, we edited the image using photo editor (just like Adobe Photoshop). The reason behind using the photo editor is mainly to increase or decrease the contrast level of brightness or to resize or rotate the image. Figure 7 shows detailed steps of image processing.

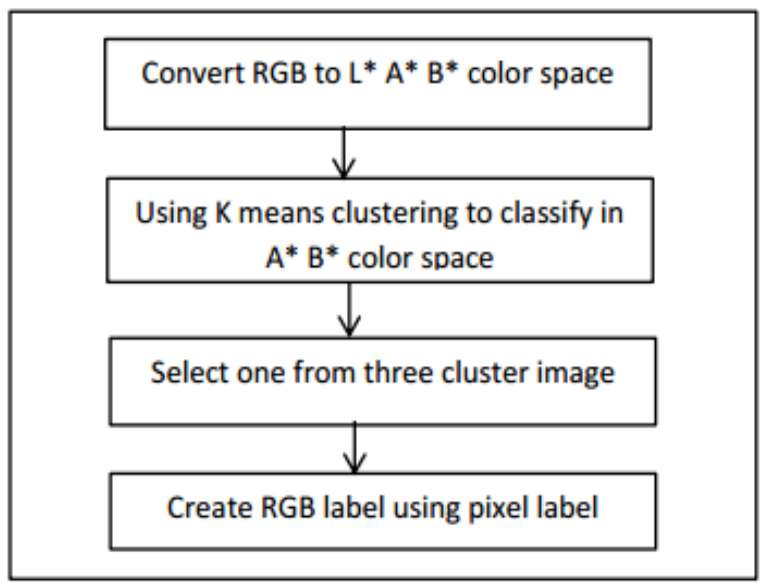

Figure 7: Steps for preprocessing images

At first, we converted the image from RGB to lab version using srgb2lab() function. It creates three images which are being resized as per their previous size. Therefore, we get three color images as follows:

i. With less brightness

ii. According to $\mathrm{A}^{*}$ (indicating where the color falls along the red-green region).

iii. According to $\mathrm{B}^{*}$ (indicating where the color falls along the blue-yellow region).
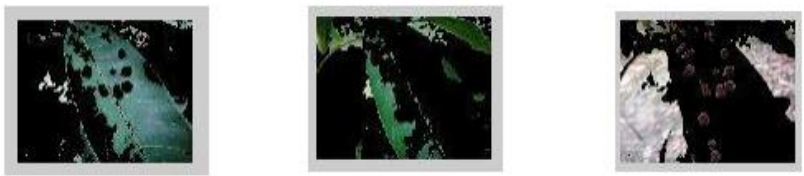

Figure 8: Steps of $A * B *$ image processing

Feature plays a major role in image processing research area. Before extracting features, diversified image preprocessing techniques like setting up contrast, correlation, measuring energy and homogeneousness are applied on the sampled image.

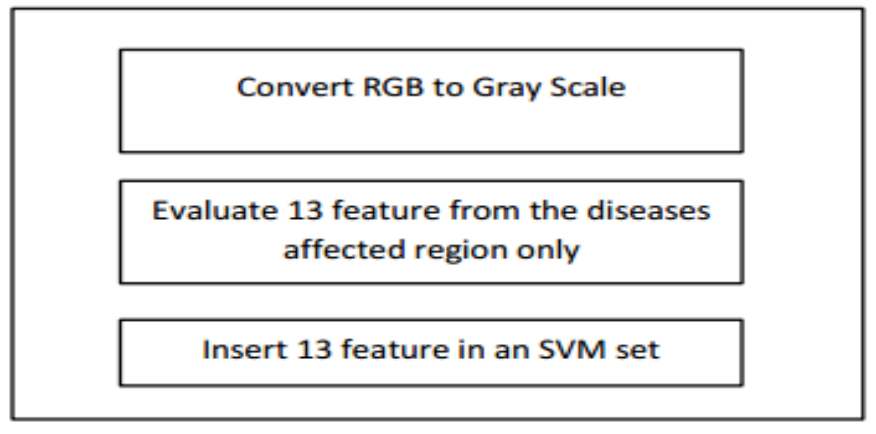

Figure 9: Steps of feature extraction

For SVM classification, at first, mat type file was loaded, which contains training datasets. After that, svmtrain(train, group) is executed on training data. Group comprises Ailment-free, Scab, Anthracnose, Sooty Mold and Red Rust. Finally, svmclassify(svmstruct, sample) classification method was run to classify. The pseudo code of SVM classification is as follows:

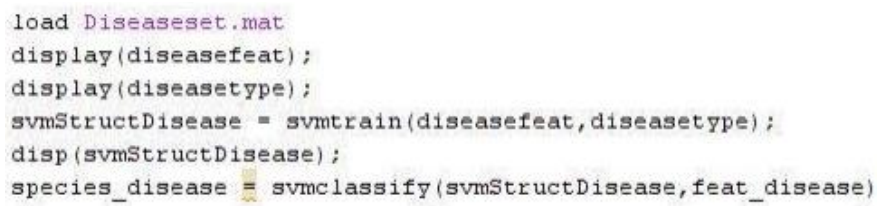

Figure 10: Pseudocode for SVM classification

\section{RESULTS AND ANALYSIS}

The classifiers are trained and tested using images of Mango leaf ailments. The sample images are divided into two halves and one half is used for training and other is used for testing and then cross-validated. The color and shape features are used to train and test neural network model. To evaluate classification performance, the experimental study focuses on comparing MLAD and SVMs that are trained by the single-step learning approach. As it is aforementioned, MLAD has been trained by 20 samples.

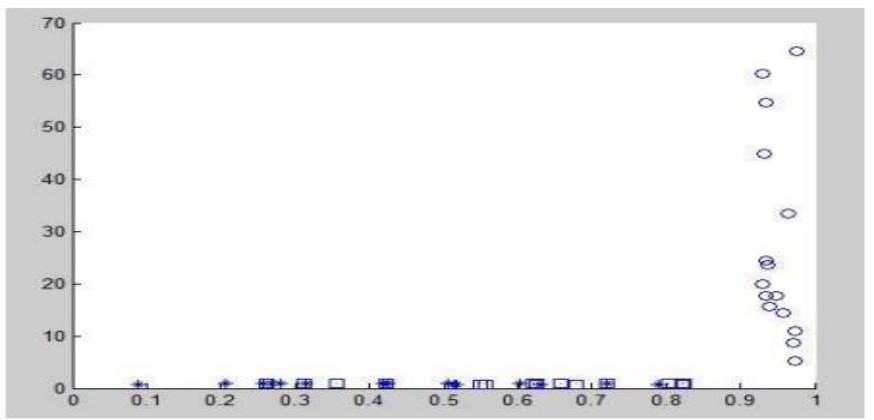

Figure 11: Classification between ailment-free data and ailment data

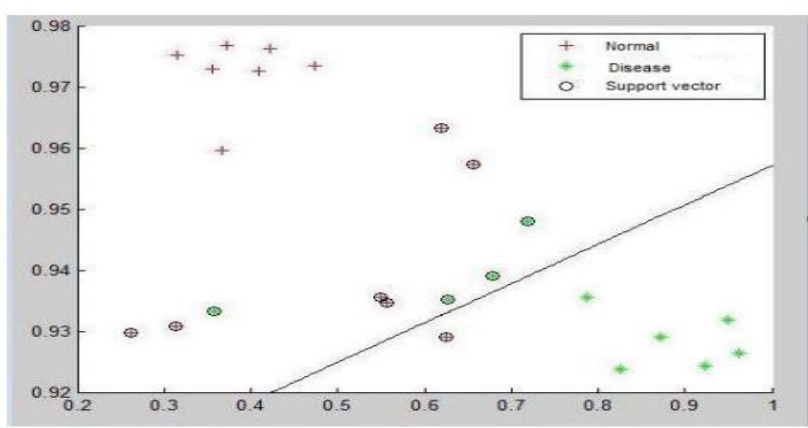

Figure 12: Using SVM classification between ailment-free data and ailment data

In figure 12 , there are two types of data, one is ailment 
free(normal) and other is ailment.

Normal data ------------- +
Ailment data ------------- *
Support vector ---------- o

The free "+" in the plot indicates the data which are not near of input data (support vector). And the adjacent of "+" and "o" are near of input data (support vector). The free "*" in the plot indicates the data which are not near of input data (support vector). And the adjacent of "**" and "o" are near of input data (support vector).

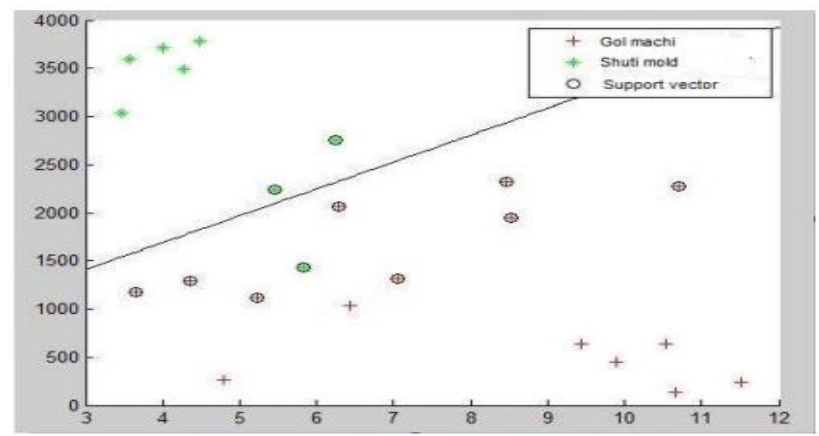

Figure 13: Using SVM classification between Scab (Bangla: Gol Machi) and Sooty Mold (Bangla: Shuti Mold) data

In figure 4.3 the following data are marked as such:

Scab (Bangla: Gol Machi) ------------------- +
Sooty Mold (Bangla: Shuti Mold) ---------
Support vector --- o

The free "+" in the plot indicates the data which are not near of input data (support vector). And the adjacent of "+" and "o" are near of input data (support vector). The free "**" in the plot indicates the data which are not near of input data (support vector). And the adjacent of "*”" and "o" are near of input data (support vector).

This system recognizes normal data which are not ailments affected is accuracy is $87.5 \%$.

By using support vector machines (SVM) we can check only two groups. Firstly, we have to check whether the input data is ailment free or not. Then using SVM we have recognized the affected ailment. That's why we have got difference accuracy for different SVM.

\section{Table 1: Performance of MLAD}

\begin{tabular}{|l|l|}
\hline \multicolumn{1}{|c|}{ Type } & \multicolumn{1}{c|}{ accuracy } \\
\hline Normal & $87.5 \%$ \\
\hline Dag rog & $75 \%$ \\
\hline Gol machi & $75 \%$ \\
\hline Moricha rog & $75 \%$ \\
\hline Shuti mold & $87.5 \%$ \\
\hline
\end{tabular}

Therefore, the average accuracy of the performance of MLAD is $(87.5+75+75+75+87.5) / 5=80 \%$.

As mentioned earlier, MLAD has been trained by 20 samples. Here, training has been continued until the error rate has reached to 2.00. To gain the better performance, 1000 training cycles were chosen for simulation.

The accuracy of classification varies from $80 \%-82 \%$ depending on the algorithms and limitations of image acquisition. Classification also is obtained with great accuracy as the case with image detection. In this case also the classification accuracy can be obtained up to $80 \%$ with correct imaging techniques and algorithms. The evaluation of processing system is increased.
For the pursuit of better generalization, SVM has been used with different NN architectures to develop MLAD.

The proposed features selection approach results in simpler, faster and easier to train Neural Network architectures, when compared to Artificial Neural Networks (ANNs) used to measure the of individual input features to the output of the neural network to get the output file of results.

For matching the ailment, several types of data were needed, such as: Left Rotate, Right Rotate, Up Rotate, Down Rotate, $100 \%, 120 \%, 140 \%, 160 \%, 180 \%, 200 \%$ and Zoom Out.

In this primary stage, four types of ailment and one type (ailment-free), in total five groups were trained. Each group comprises four different types of images. For achieving more accuracy, more images should be added in each group.

As accuracy depends on training data, this work has 20 training data with 13 features. For classification, SVM classifier has been used.

\section{CONCLUSION}

Bangladesh is an Agriculture-based country. Its economy primarily depends on Agriculture. It is a National challenge for Agricultural Scientists and Researchers to work day and night to monitor and improve the food grains. It is very difficult to infer the varieties of a leaf ailment by simple visual observation as well. It is also time consuming and can be accomplished by the trained botanists. So, MLAD plays the vital automated role.

In the near future, investigation would be continued to reform the potential effectiveness and ability the proposed method described in this paper. Some other experimental study could be designed to observe the influence of MLAD in other sectors.

Future work will include research along two directions:

$$
\begin{aligned}
& \text { i. Comparing textures base features } \\
& \text { ii. Color features for improving recognition } \\
& \begin{array}{l}
\text { accuracies. }
\end{array}
\end{aligned}
$$

An option could be used for future consideration is to use texture, which has been beyond range in this work. However, the process of recognition should not be hampered for implementing new features.

\section{ACKNOWLEDGMENT}

Sincere appreciation goes to Mr. Akm Ashiquzzaman, Teaching Assistant, Department of Computer Science and Engineering, University of Asia Pacific, Dhaka, Bangladesh for his technical assistance and endless support towards this research work.

\section{REFERENCES}

[1] Anand Swaroop, Sidney J. Stohs, Manashi Bagchi, Hiroyoshi Moriyama, and Debasis Bagchi. "Mango (Mangifera indica Linn) and Anti-Inflammatory Benefits: Versatile Roles in Mitochondrial Bio-Energetics and Exercise Physiology." Functional Foods in Health and Ailment 8, no. 5 (2018): 267-279.

[2] K. Srunitha and D. Bharathi. "Mango Leaf Unhealthy Region Detection and Classification." In Computational Vision and Bio Inspired Computing, pp. 422-436. Springer, Cham, 2018..

[3] A. Camargo and J. S. Smith. "An image-processing based algorithm to automatically identify plant ailment visual symptoms." Biosystems engineering 102, no. 1 (2009): 921.

[4] Bikash Chandra Karmokar, Mohammad Samawat Ullah, Md Kibria Siddiquee, and Kazi Md Rokibul Alam. "Tea leaf ailments recognition using neural network ensemble." International Journal of Computer Applications 114, no. 17 
(2015).

[5] Panagiotis Tzionas, Stelios E. Papadakis, and Dimitris Manolakis. "Plant leaves classification based on morphological features and a fuzzy surface selection technique." In Fifth international conference on technology and automation, Thessaloniki, Greece, pp. 365-370. 2005.

[6] Michael Egmont-Petersen, Dick de Ridder, and Heinz Handels. "Image processing with neural networks-a review." Pattern recognition 35, no. 10 (2002): 2279-2301.

[7] Sladojevic, Srdjan, Marko Arsenovic, Andras Anderla, Dubravko Culibrk, and Darko Stefanovic. "Deep neural networks based recognition of plant ailments by leaf image classification." Computational intelligence and neuroscience 2016.

[8] Melissa B. Riley, Margaret R. Williamson, and Otis Maloy. "Plant ailment diagnosis." The plant health instructor (2002)

[9] Nur Badariah Ahmad Mustafa, Syed Khaleel Ahmed, Zaipatimah Ali, Wong Bing Yit, Aidil Azwin Zainul Abidin, and Zainul Abidin Md Sharrif. "Agricultural produce sorting and grading using support vector machines and fuzzy logic." In Signal and Image Processing Applications (ICSIPA), 2009 IEEE International Conference on, pp. 391-396. IEEE.

[10] Imran Maqbool, Salman Qadri, Dost Muhammad Khan, and Muhammad Fahad. "Identification of Mango Leaves by Using Artificial Intelligence." International Journal of Natural and Engineering Sciences 9, no. 3 (2015): 45-53.

[11] Maureen Caudill, "Neural networks primer, part VIII." AI Expert 4, no. 8 (1989): 61-67.

[12] Egmont-Petersen, Michael, W. R. M. Dassen, and Johan HC Reiber. "Sequential selection of discrete features for neural networks-A Bayesian approach to building a cascade." Pattern Recognition Letters 20, no. 11-13 (1999): 1439-1448.

[13] Gaurav Kumar and Pradeep Kumar Bhatia. "A detailed review of feature extraction in image processing systems." In Advanced Computing \& Communication Technologies (ACCT), 2014 Fourth International Conference on, pp. 512. IEEE, 2014.

[14] Dong ping Tian, "A review on image feature extraction and representation techniques." International Journal of Multimedia and Ubiquitous Engineering 8, no. 4 (2013): 385-396.

[15] Dengsheng Zhang and Guojun Lu, "Review of shape representation and description techniques." Pattern recognition 37, no. 1 (2004): 1-19.

[16] Devashish Pujari, Rajesh Yakkundimath, and Abdulmunaf S. Byadgi. "SVM and ANN based classification of plant ailments using feature reduction technique." IJIMAI 3, no. 7 (2016): 6-14.

[17] Yuan Tian, Chunjiang Zhao, Shenglian Lu, and Xinyu Guo. "SVM-based multiple classifier system for recognition of wheat leaf ailments." In World Automation Congress (WAC), 2012, pp. 189-193. IEEE, 2012.

[18] Santanu Phadikar and Jaya Sil. "Rice ailment identification using pattern recognition techniques." In Computer and Information Technology, 2008. ICCIT 2008. 11th International Conference on, pp. 420-423. IEEE, 2008.
[19] Ajay A. Gurjar and Viraj A. Gulhane. "Ailment detection on cotton leaves by eigenfeature regularization and extraction technique." International Journal of Electronics, Communication and Soft Computing Science \& Engineering (IJECSCSE) 1, no. 1 (2012): 1 .

[20] Rakesh Kaundal, Amar S. Kapoor, and Gajendra PS Raghava. "Machine learning techniques in ailment forecasting: a case study on rice blast prediction." BMC bioinformatics 7, no. 1 (2006): 485 .

[21] MS Prasad Babu and B. Srinivasa Rao. "Leaves recognition using back propagation neural network-advice for pest and ailment control on crops." IndiaKisan. Net: Expert Advisory System (2007).

[22] Isabelle Guyon and André Elisseeff. "An introduction to feature extraction." In Feature extraction, pp. 1-25. Springer, Berlin, Heidelberg, 2006.

[23] Melville and Mooney, "Creating Diverse Ensemble Classifiers to Reduce Supervision", PhD Thesis, Department of Computer Sciences, University of Texas at Austin, November 2005

[24] Hafiz T. Hassan, Muhammad U. Khalid, and Kashif Imran. "Intelligent Object and Pattern Recognition using Ensembles in Back propagation neural network." International Journal of Electrical \& Computer Sciences (IJECS-IJENS) Vol 10 (2010).

[25] Robi Polikar, "Ensemble based systems in decision making." IEEE Circuits and systems magazine 6 , no. 3 (2006): 21-45.

[26] Philipp, Isabelle, and Thomas Rath. "Improving plant discrimination in image processing by use of different colour space transformations." Computers and electronics in agriculture 35 , no. 1 (2002): 1-15

[27] Jagadeesh D. Pujari, Rajesh Yakkundimath, and Abdulmunaf S. Byadgi. "Recognition and classification of normal and affected agriculture produce using reduced color and texture features." International Journal of Computer Applications 93, no. 11 (2014).

[28] Jagadeesh D. Pujari, Rajesh Yakkundimath, and Abdulmunaf Syedhusain Byadgi. "Automatic fungal disease detection based on wavelet feature extraction and PCA analysis in commercial crops." International Journal of Image, Graphics and Signal Processing 6, no. 1 (2013): 24-31.

[29] P S. Landge, Patil S A., Khot D S., Otari O D. and Malavkar U G, Automatic Detection and Classification of Plant Ailment through Image Processing, International Journal of Advanced Research in Computer Science and Software Engineering, Vol.3, Issue.7, July 2013, pp.798801.

[30] S. Prasad, Piyush Kumar., Hazra R. and Ajay Kumar, Plant Leaf Ailment Detection Using Gabor Wavelet Transform, Proceedings of 3rd International Conference on Swarm, Evolutionary, and Memetic Computing, Springer-Verlag, Berlin, Heidelberg, 2012

[31] J D Pujari, Content Based Image Retrieval Using Color and Texture Features, Ph.D. Thesis, Gulburga University, Gulburga, 2008

[32] A N. Rathod, Tanawal B. and Shah V, Image Processing Techniques for Detection of Leaf Ailment, International 
Journal of Advanced Research in Computer Science and Software Engineering, Vol.3, Issue.11, November 2013, pp.397-399.

[33] N. Razmjooya, Mousavib B S. and Soleymani F, A Real Time Mathematical Computer Method for Potato Inspection Using Machine Vision, Computers and Mathematics with Applications, Vol. 63, 2012, pp.268-279.

[34] Savakar D G. and Anami B S, Improved Method for Identification and Classification of Foreign Bodies Mixed Food Grains Image Samples, International Journal of Artificial Intelligence and Machine Learning, Vol.9, Issue.1, 2009, pp.1-8.

[35] Sungkur R K., Baichoo S. and Poligadu A, 2013, An Automated System to Recognize Fungi-caused Ailments on Sugarcane Leaves, Proceedings of Global Engineering, Science and Technology Conference, Singapore, 3-4 October, 2013

[36] Vibhute A. and Bhode S K, Applications of Image Processing inAgriculture: A Survey, International Journal of Computer ApplicationsVol. 52, Issue. 2, August 2012, pp.34-40.

[37] Dheeb Al Bashish, Malik Braik, and Sulieman BaniAhmad, "A Framework for Detection and Classification of Plant Leaf and Stem Ailments," Department of Information Technology, IEEE International Conference on Signal and Image zProcessing, 2010

[38] S. Ananthi, S. Vishnu Varthini, "Detection and classification of plant leaf ailments,"International Journal of
Research in Engineering \& Applied Sciences, Volume 2, Issue 2 (February 2012), ISSN: 2249-3905, 2012.

[39] Jiazhi Pan, Yong He, "Recognition of plants by leaves digital image and neural network," International Conference on Computer Science and Software Engineering,IEEE Computer Society, 2008.

[40] Xinhong Zhang, Fan Zhang, "Images Features Extraction of Tobacco Leaves,"Congress on Image and Signal Processing, IEEE Computer Society, 2008.

[41] A. Meunkaewjinda, P. Kumsawat, K. Attakitmongcol and A. Srikaew, "Grape leaf ailment detection from color imagery using hybrid intelligent system," IEEE Proceedings of ECTI-CON, 2008.

[42] Zulkifli Bin Husin, Abdul Hallis Bin Abdul Aziz, Ali Yeon Bin Md Shakaff, Rohani Binti S Mohamed Farook, "Feasibility Study on Plant Chili Ailment Detection Using Image Processing Techniques," Third International Conference on Intelligent Systems Modelling and Simulation, IEEE Computer Society, 2012

[43] Panagiotis Tzionas, Dimitris Manolakis, Stelios E. Papadakis "Plant leaves classification based on morphological features and a fuzzy surface selection technique."

[44] Dr. V.S.Rajpurohit, Shruti Bhat, Shreya Devurkar, Shubhangi Patil, Supriya Sirbi, "Application of Image Processing Techniques in Object Shape Recognition",International Journal of Latest Trends in Engineering and Technology (IJLTET), 2013. 